\title{
Aurora-A gene is frequently amplified in basal-like breast cancer
}

\author{
SYNNÖVE STAFF $^{1}$, JORMA ISOLA ${ }^{1}$, MERVI JUMPPANEN ${ }^{2}$ and MINNA TANNER ${ }^{1,3}$ \\ ${ }^{1}$ Laboratory of Cancer Biology, Institute of Medical Technology, University and University Hospital of Tampere; \\ ${ }^{2}$ Department of Pathology, Seinäjoki Central Hospital, Seinäjoki; ${ }^{3}$ Department of Clinical Oncology, \\ University Hospital of Tampere, Tampere, Finland
}

Received May 19, 2009; Accepted June 15, 2009

DOI: 10.3892/or_00000637

\begin{abstract}
Aurora-A is a serine-threonine kinase having vital cellular functions in mitosis and is a promising target for therapy in treating patients with cancer. This study assesses the clinicopathological associations of AURKA gene amplification in clinical breast tumors and association of gene copy number with its mRNA and protein expression in breast cancer cell lines. In this pilot study, we examined Aurora-A gene $(A U R K A)$ amplification in 126 clinical breast tumors by chromogenic in situ hybridisation (CISH). AURKA amplification (found in $21 \%$ ) showed an association with basal-like tumor phenotype $(\mathrm{p}=0.046)$. A separate series of basal-like breast tumors $(\mathrm{n}=26)$ provided further evidence of the importance of AURKA in these tumors. AURKA amplification status was associated with immunohistochemically detectable Aurora-A protein expression $(\mathrm{p}<0.0001)$. In breast cancer cell lines, gene amplification was strongly associated with high mRNA expression $(\mathrm{p}<0.0001)$. JIMT-1 cell line was found as a possible in vitro model system for testing Aurora-A inhibitors, since it has been classified as basal-like breast cancer and here it showed both AURKA gene amplification and elevated mRNA expression. AURKA gene amplification is a common genetic aberration in breast cancer, especially in tumors displaying basal-like phenotype. Thus, these patients might be suitable candidates for future targeted therapies with Aurora-A inhibitors.
\end{abstract}

Correspondence to: Dr Synnöve Staff, Laboratory of Cancer Biology, Institute of Medical Technology, University and University Hospital of Tampere, Biokatu 6, 33014 Tampere, Finland E-mail: synnove.staff@uta.fi

Abbreviations: AURKA, Aurora Kinase A gene; CISH, chromogenic in situ hybridization; ER, estrogen receptor; FISH, fluorescence in situ hybridization; IHC, immunohistochemistry; PgR progesterone receptor; qRT-PCR, quantitative reverse transcriptase polymerase chain reaction; TMA, tissue microarray

Key words: Aurora-A, basal-like phenotype, breast cancer, gene amplification, chromogenic in situ hybridization

\section{Introduction}

Amplification of chromosomal region $20 \mathrm{q}$ has been reported to occur frequently in breast cancer as well as in many other cancer types $(1,2)$. One of the identified candidate oncogenes in this region is AURKA (also named as BTAK or $S T K 15$ or AIK1) (3), which encodes the serine-threonine kinase Aurora-A involved in controlling centrosome function, mitotic spindle formation and proper chromosome segregation during mitosis (4). AURKA may function as an oncogene, since its overexpression has been shown to lead to centrosome amplification, aneuploidy and transformation of mammalian cells (5). The essential function of Aurora-A in cell division suggests it to be a potential drug target in cancer therapy $(6,7)$. In fact, Aurora-A inhibitors have been shown to inhibit tumor growth in xenograft models $(8,9)$ and some molecules have already entered phase I and II clinical studies (10). However, at present there are no clinical biomarkers for Aurora-A inhibitors in breast cancer or any other cancer types.

This pilot study assesses the clinicopathological associations of AURKA gene amplification in clinical breast tumors. In addition, Aurora-A mRNA expression, gene amplification and DNA sequence variation are further characterized in a set of breast cancer cell lines.

\section{Materials and methods}

Clinical tumor specimens and breast cancer cell lines. After receiving ethical permission, the study material consisted of a cohort of 126 consecutive primary invasive ductal breast carcinomas with tumor samples available and was obtained from Seinäjoki District Hospital, Seinäjoki, Finland. Histological typing, grading, staging and evaluation of estrogen receptor $(\mathrm{ER})$ and progesterone receptor $(\mathrm{PgR})$ status were done as part of routine diagnostic protocol according to standard histopathologic techniques and WHO classification. Formalin-fixed, paraffin-embedded tumor samples were then used for preparation of tissue microarrays (TMA). Detailed information on preparation of TMA blocks is available on request.

Breast cancer cell lines BT-474, MCF-7, MDA-MB-453, MDA-MB-134-VI, MDA-MB-361, SK-BR-3, UACC-812, UACC-893, ZR-75-1, ZR-75-30, CAMA-1 and DU4475 were obtained from The American Type Culture Collection. 


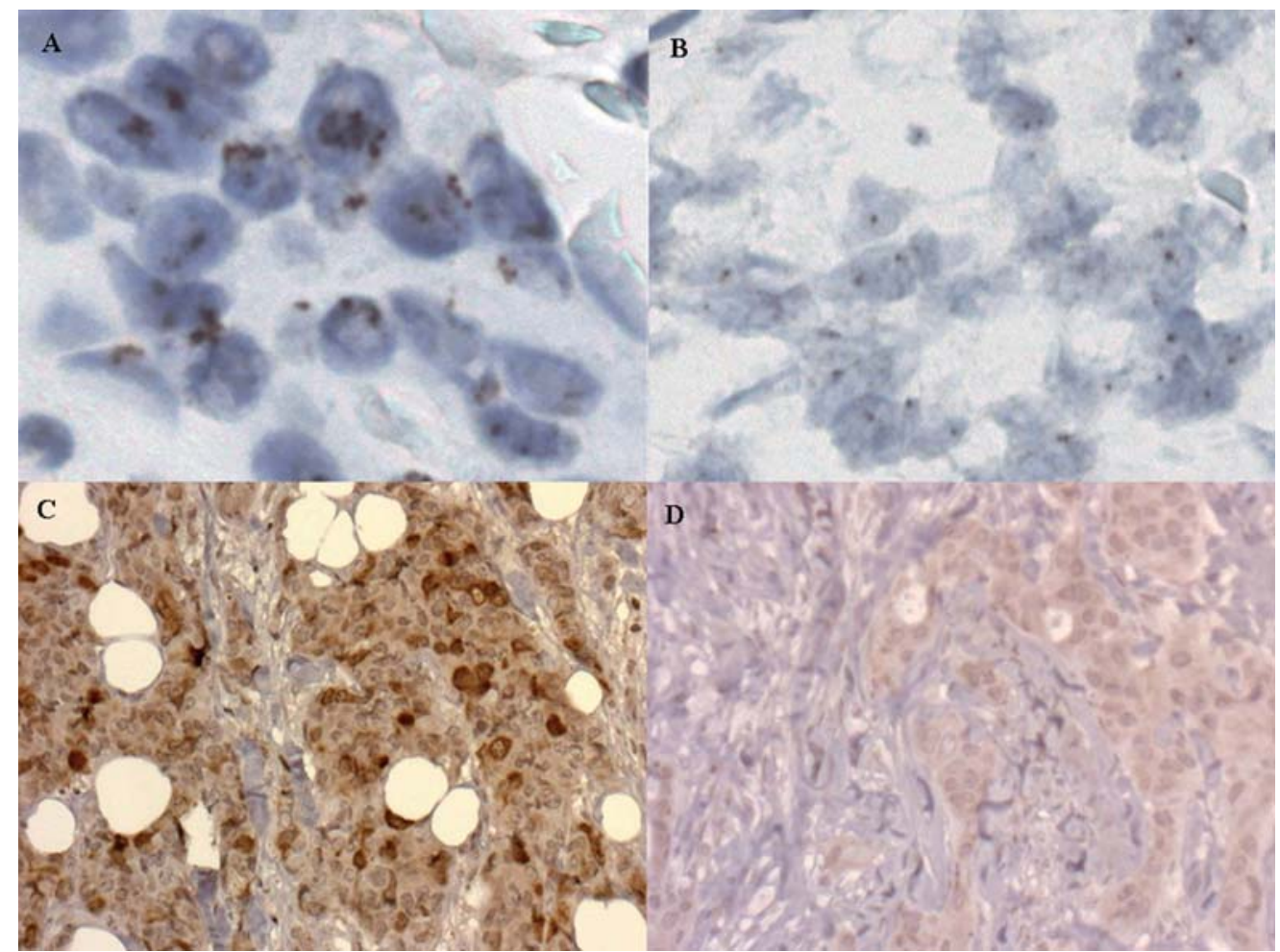

Figure 1. A tissue tumor array image showing amplification (A) and normal copy number (B) of AURKA gene by CISH. A tissue tumor array image showing positive (C) and negative (D) immunohistochemical staining of Aurora-A antibody (NCL-L-AK2).

Breast cancer cell line L56Br-C1 deficient in BRCA1 gene product was kindly provided by Professor Åke Borg, University of Lund, Sweden (11). Breast cancer cell line clinically resistant to trastuzumab (Herceptin) JIMT-1 was established and characterized in our laboratory (12) and is available via DSMZ (www.dsmz.de). All the breast cancer cell lines used in this study were cultured in recommended conditions and DNA, RNA and protein extraction were done using standard procedures (11).

In situ hybridization analyses. A PAC (P1 artificial chromosome) probe specific for the AURKA gene on chromosome 20q13 (PAC clone 189F4) and a reference probe to AURKA, a BAC (bacterial artificial chromosome) clone RP11-96L6 (a unique sequence probe located at 20p11.1-11.22) were used in the hybridization analyses. Locus 20p11.1-11.22 specific probe RP11-96L6 was used as a reference since specific chromosome 20 centromere probes are not available.

Chromogenic in situ hybridization (CISH) was carried out as previously described (13). Briefly, TMA slides were deparaffinized and incubated in citric acid buffer $(\mathrm{pH} \mathrm{6.0)}$ at $80^{\circ} \mathrm{C}$ for $1 \mathrm{~h}$, followed by cooling at room temperature. Enzymatic digestion was carried out by applying $100 \mu \mathrm{l}$ of pepsin to the slides (Digest-All III solution; Zymed, Inc., San Francisco, CA). After dehydration, a digoxigenin-labelled PAC probe specific for AURKA gene (PAC clone 189F4) or the reference probe (clone RP11-96L6) was applied to the slides. The sections were denatured on a thermal plate and hybridization was carried out overnight at $37^{\circ} \mathrm{C}$. The probes were detected by consecutive incubations with mouse antidigoxigenin (Roche Biochemicals, Mannheim, Germany), anti- mouse-peroxidase polymer (Powervision ${ }^{+}$; Immunovision, Inc.) and $\mathrm{DAB}$ chromogen. The sections were then counterstained with hematoxylin and embedded.

The fluorescence in situ hybridization (FISH) was carried out as previously described (14). The probe specific for the AURKA gene (PAC 189F4) and the reference probe (clone RP11-96L6) were labelled by nick-translation. Slides were hybridized with $A U R K A$ or RP11-96L6 probe as previously described (14). In CISH and FISH analyses gene amplification of AURKA was defined as the ratio of AURKA/RP1196L6 signals $\geq 2$.

Immunohistochemistry (IHC). The specificity of the primary antibody for Aurora-A (NCL-L-AK2, Novocastra Laboratories, Newcastle, UK) was verified using cell line protein lysates by standard Western blotting. The NCL-L-AK2 antibody detects specifically one band migrating at $45 \mathrm{kDa}$ (data not shown). Before tumor specimen analyses the Aurora-A antibody NCL-L-AK2 was tested on tissue sections of normal breast and colon. Immunohistochemical assays on TMA block sections were then carried out with primary Aurora-A antibody as previously described (15). Using an arbitrary cutoff, a strong, clearly positive staining pattern was classified as positive. No or faint staining pattern undetected by IHC digital image software was classified as negative. The software was written using ImageJ (http://rsb.info.nih.gov/ij/) and is based on the colour deconvolution principle as previously described (16).

The cohort of tumors of this study has been previously analyzed for cytokeratin 5/14 (CK5/14) immunostaining pattern reflecting the basal tumor phenotype. A CK5/CK14 antibody cocktail was used in immunostaining analyses. 
Tumor specimens were classified as positive (basal phenotype) if $>20 \%$ of the neoplastic cells showed cytoplasmic staining by this CK5/CK14 antibody cocktail (17).

DNA sequencing and quantitative reverse transcriptasepolymerase chain reaction ( $q R T-P C R)$. In order to study whether genomic mutations of $A U R K A$ are present in breast cancer cell lines, all exons (exons 1-9) were amplified using a standard PCR protocol. The oligonucleotide primers and specific PCR reaction conditions for each primer pair are available on request. Direct sequencing of PCR products was done using BigDye3 termination chemistry (Applied Biosystems, Foster City, CA) and an ABI 310 or 3100 genetic analyzer (Applied Biosystems) according to the manufacturer's instructions.

AURKA gene expression was studied using total RNA from breast cancer cell lines, which was reverse transcribed using Superscript II reverse transcriptase and random hexamer primer (Invitrogen, Life Technologies, USA). Primer and probe sequences were designed for Aurora-A and Cyclin B1, which was used as a control. Since the expression of Aurora-A is known to be cell cycle-dependent (18), we used Cyclin B1 as a reference gene due to its cell cycle dependence to adjust for the variation in the tumor proliferation rate (19). The primer and probe sequences are available on request. The quantitative real-time RT-PCR reactions were performed with the Light Cycler (LC) (20) and the Light Cycler software was used in the analyses as previously described (14). The quantitative real-time RT-PCR assays were repeated twice, and variation between runs was $<15 \%$. Detailed reaction conditions are available on request.

\section{Results}

Gene amplification by CISH and protein expression by IHC in clinical breast tumors. Of the 126 primary sporadic breast tumors, $26(21 \%)$ showed AURKA gene amplification by CISH (Fig. 1A). AURKA gene amplification showed an association with basal-like tumor phenotype (Pearson $\chi^{2}$, $\mathrm{p}=0.046$ ) and a tendency towards PgR negativity (Pearson $\chi^{2}$, $\mathrm{p}=0.086)$. In order to study correlation between AURKA gene amplification and basal tumor phenotype in more detail, we analyzed an additional series of 26 primary invasive ductal breast carcinomas of basal-like tumor pheno-type (i.e. showing positive cytokeratin $5 / 14$ immunostaining). In this series 8/26 (31\%) tumors showed AURKA gene amplification. Cross-tabulation of AURKA gene copy number status with clinicopathological characteristics is shown in Table I.

Aurora-A protein expression was analyzed by immunohistochemistry $(\mathrm{n}=97)$. Of these, $23(24 \%)$ were positive and $74(76 \%)$ were negative for Aurora-A antibody staining (Fig. 1C and D). Aurora-A immunostaining pattern associated statistically significantly with AURKA gene amplification status (Pearson $\chi^{2}, \mathrm{p}<0.0001$; Table II).

Aurora-A genomic sequence, gene copy number and mRNA expression in breast cancer cell lines. All exons of AURKA gene were sequenced from 12 breast cancer cell lines. No exonic mutations were found. Coding region polymorphism T91A (or 449t/a), resulting in amino acid substitution (F31I)
Table I. Clinicopathological association of AURKA gene amplification by CISH in breast tumors.

\begin{tabular}{|c|c|c|}
\hline Variable & $\begin{array}{c}\text { Gene } \\
\text { amplification }^{\mathrm{a}}(\%)\end{array}$ & P-value \\
\hline All tumors & $26 / 126(21)$ & - \\
\hline \multicolumn{3}{|c|}{ Histological grade } \\
\hline I or II & $13 / 75(13)$ & 0.267 \\
\hline III & $13 / 51(25)$ & \\
\hline \multicolumn{3}{|c|}{ Estrogen receptor } \\
\hline Negative & $6 / 23(26)$ & 0.475 \\
\hline Positive & 20/103 (19) & \\
\hline \multicolumn{3}{|c|}{$\begin{array}{l}\text { Progesterone } \\
\text { receptor }\end{array}$} \\
\hline Negative & $10 / 32(31)$ & 0.086 \\
\hline Positive & $16 / 94(17)$ & \\
\hline \multicolumn{3}{|c|}{$\begin{array}{l}\text { No. of lymph node } \\
\text { metastasis }\end{array}$} \\
\hline 0 & $15 / 71(21)$ & 0.877 \\
\hline$\geq 1$ & $11 / 55(20)$ & \\
\hline \multicolumn{3}{|c|}{ Age at diagnosis } \\
\hline$<50$ & $6 / 28(21)$ & 0.906 \\
\hline$\geq 50$ & 20/98 (20) & \\
\hline \multicolumn{3}{|c|}{ HER2 amplification ${ }^{\mathrm{c}}$} \\
\hline No & 19/100 (19) & 0.576 \\
\hline Yes & $6 / 25(24)$ & \\
\hline \multicolumn{3}{|c|}{$\begin{array}{l}\text { Basal tumor phenotype } \\
\text { (positive cytokeratin } 5 / \\
14 \text { immunostaining) }\end{array}$} \\
\hline Negative & 19/109 (17) & 0.046 \\
\hline Positive & $5 / 12(42)$ & \\
\hline
\end{tabular}

${ }^{\mathrm{a}}$ Gene amplification of $A U R K A$ was recorded when the ratio of AURKA signals compared to RP11-96L6 signals at 20p11.1-11.22

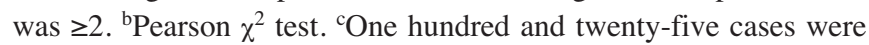
available for association analysis of HER2 and AURKA gene amplification and 121 cases for association analysis of cytokeratin 5/14 (CK5/14) immunostaining pattern and AURKA gene amplification.

was found in five cell lines either as a homozygous AA (UACC-812, BT-474, ZR-75-30) or heterozygous TA allelic variant (L56Br-C1 and CAMA-1).

$A U R K A$ gene copy number in the cell lines was analyzed by FISH $(n=13)$. Gene amplification was detected in $6 / 13$ (46\%) of the cell lines, of which high level AURKA amplification (defined as $A U R K A /$ reference probe copy number ratio $>2$ ) was found in BT-474 and SK-BR-3 $(2 / 13 ; 15 \%)$ and low level $A U R K A$ amplification (defined as AURKA/reference probe copy number ratio $\sim 2$ ) was found in four cell lines JIMT-1, MCF-7, UACC-812 and UACC-893 (4/13; 31\%; 
Table II. The cross-tabulation of AURKA gene copy number status with Aurora-A (NCL-L-AK2)a immunohistochemistry.

$\begin{array}{cc}\text { Gene } & \text { No relative gene copy } \\ \text { amplification }^{\mathrm{b}}(\%) & \text { number change }(\%)\end{array}$

Positive staining for

$12 / 20(60)$

$11 / 77$ (14)

AURORA-A antibody (NCL-L-AK2)

Negative staining for AURORA-A

antibody (NCL-L-AK2)

$8 / 20(40)$

66/77 (86)

aAurora-A immunostaining pattern associated statistically significantly with AURKA gene amplification status (p<0.0001, $\chi^{2}$ test). ${ }^{\mathrm{b}} \mathrm{Gene}$ amplification of AURKA was recorded when the ratio of AURKA signals compared to RP11-96L6 signals at 20p11.1-11.22 was $\geq 2$.

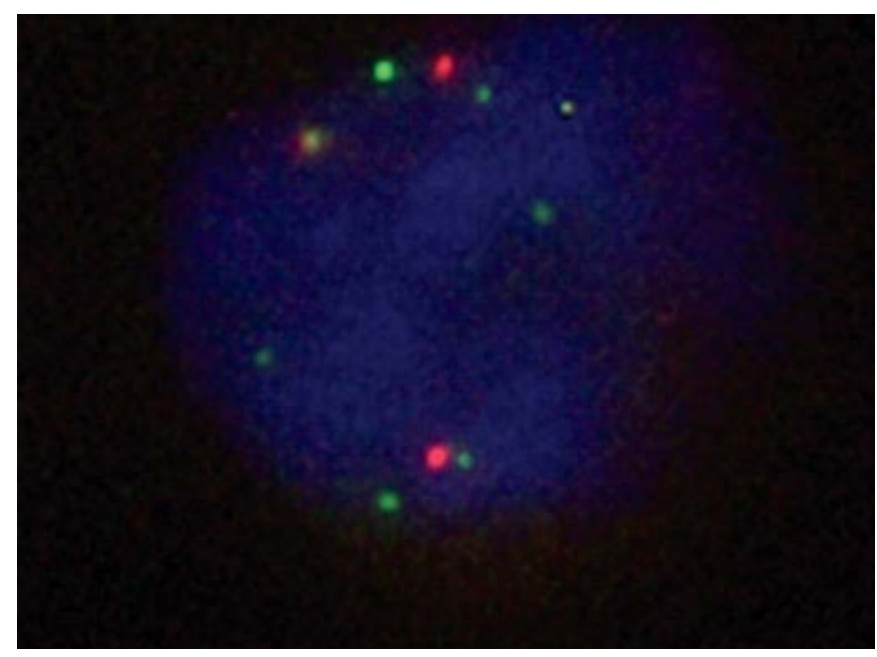

Figure 2. Dual-colour FISH image of JIMT-1 cell line showing an amplification of AURKA (green signals), compared to reference probe (RP11-96L6 at 20p11.1-11.22; red signals).

Fig. 2). Aurora-A mRNA expression correlated significantly with gene copy number, but not with T91A polymorphism genotype $(p<0.0001$ and $p=0.473$, respectively; Fig. 3$)$.

\section{Discussion}

In previous studies of breast cancer Aurora-A protein overexpression has been shown to correlate with aggressive clinicopathological parameters such as hormone receptor negativity and high histological grade $(21,22)$. In our study a trend between AURKA gene amplification and progesterone receptor negativity was also seen. Interestingly, we showed that AURKA gene amplification was found in a significant proportion of tumors displaying basal-like tumor phenotype. Basal-like breast tumors account for approximately 10-15\% of all breast cancers, characteristically express high-molecular weight basal cytokeratins and are considered as clinically aggressive tumors (23). Amplification at $20 \mathrm{q}$ is a common chromosomal change in hereditary BRCA1 and BRCA2 breast and ovarian cancers $(24,25)$ and $A U R K A$ gene amplification has been associated with breast tumors harbouring a $B R C A 2$ mutation (26). These hereditary tumors, especially those in
BRCAl mutation carriers, are generally considered to belong to a basal-like subtype of breast cancer (17). AURKA gene amplification, however, has not been correlated with basallike phenotype in sporadic breast tumors before. This novel finding may be of clinical importance, since basal-like breast tumors are aggressive and usually lack conventional therapeutic targets, such as hormone receptors or HER-2 $(17,27)$. Based on the present study, patients with basal-like breast cancer might be candidates for future studies with Aurora-A inhibitors.

Among the cell lines analyzed in this study, in theory, JIMT-1 is a promising model system for testing the Aurora-A inhibitor molecules in basal-like tumors. Despite its paradoxical HER-2 positivity, JIMT-1 has constantly been classified as basal-like, mainly based on its strong overexpression of basal cytokeratins CK 5 and 14 (12). In our study, JIMT-1 had additional resemblance to clinical basal-like tumors by showing both AURKA amplification and overexpression of Aurora-A mRNA. Since AURKA gene amplification is a more common phenomenon in breast cancer than basal-like pheno-type per se, it was expected to find AURKA gene amplification also in cell lines not filling criteria for basallike phenotype. This was true regarding breast cancer cell lines such as BT-474, SK-BR-3, MCF-7 and UACC-812 (28). However, our results are in line with previously described studies showing AURKA gene amplification in the cell lines BT-474, SK-BR-3 and MCF-7 (3).

$A U R K A$ has been identified as one of the genes undergoing amplification in breast cancer already many years ago (3). The association between gene amplification and overexpression in breast cancer, however, has remained contradictory $(3,5)$. Our data obtained from breast cancer cell lines showed that AURKA gene amplification correlates strongly with Aurora-A mRNA level. We aimed at adjusting Aurora-A expression for differences in cell proliferation, which is a major determinant of Aurora-A mRNA levels (18). In the present study, we showed that AURKA gene amplification associated with increased mRNA expression in breast cancer cell lines. In clinical tumors, gene amplification status was also associated with Aurora-A expression pattern. Gene amplification seemed to be the most significant factor explaining overexpression since positive staining was seen only in $14 \%$ of tumors in the absence of gene amplification. AURKA gene 


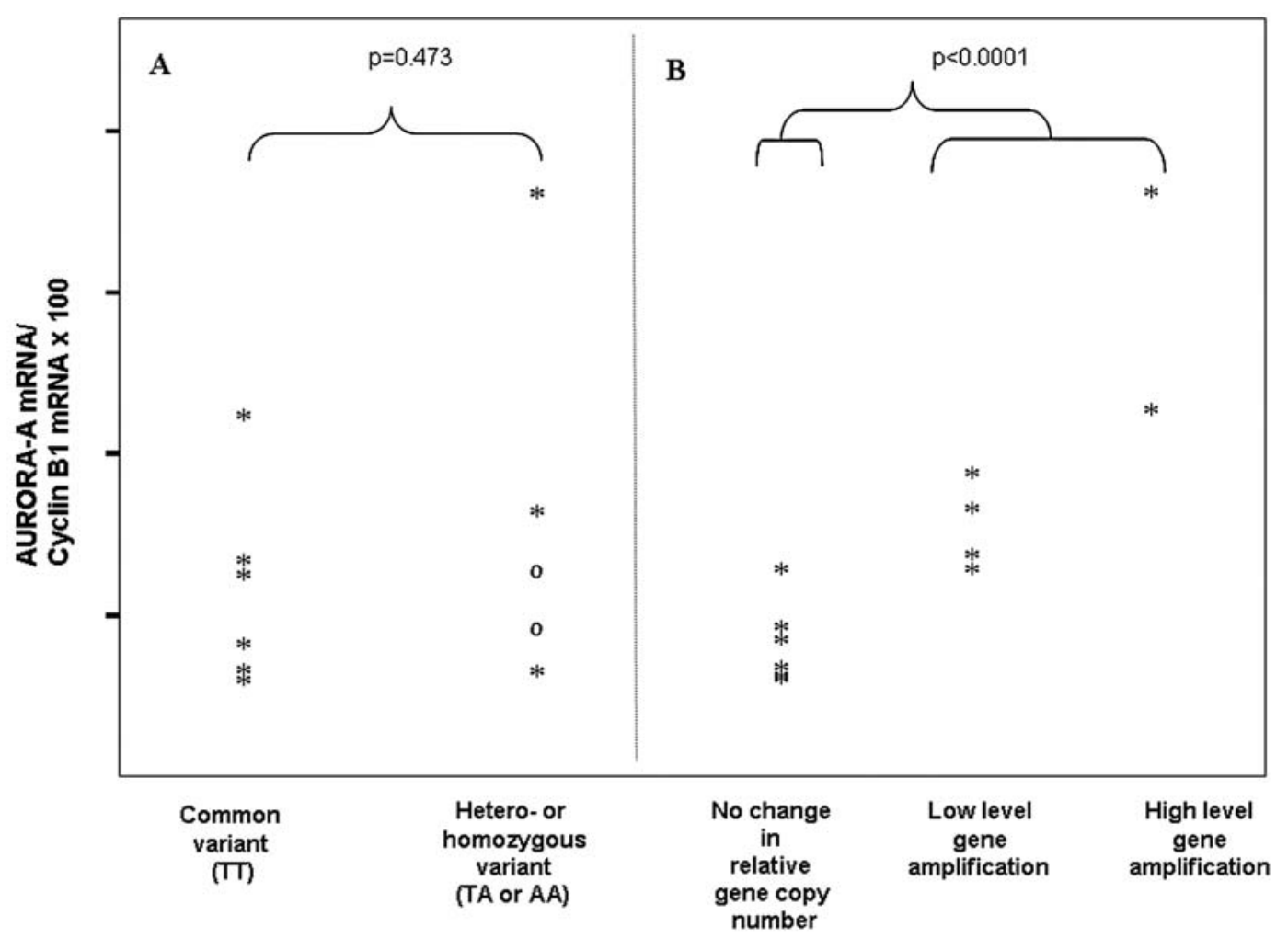

Figure 3. (A) Relative expression levels of Aurora-A mRNA analyzed by real-time RT-PCR in breast cancer cell lines according to T91A genotype. Heterozygote (TA, shown by open circles) and homozygote (AA) variants are grouped together. (B) Relative expression levels of Aurora-A mRNA analyzed by real-time RT-PCR in breast cancer cell lines according to AURKA gene amplification status. In statistical analyses cell lines with low level (AURKA/ reference probe copy number $=2)$ and high level $(A U R K A /$ reference probe copy number $>2)$ gene amplification were grouped together and were compared to cell lines with no relative gene copy number change.

amplification was not always (40\%) associated with positive immunostaining, and this finding can be partly explained by the complexity of $20 \mathrm{q}$ amplicon, the possible confounding effect of cell proliferation and the qualitative nature of immunohistochemistry.

Thus, strength of the present study is the use of a chromosome-arm specific reference probe, which made it possible to distinguish between true AURKA amplification and general polyploidy at 20q. To date it is well established that breast cancer tumors carry complex chromosomal rearrangements at $20 \mathrm{q}$, often involving genes other than AURKA (29). Limitations of the study include a relatively small clinical tumor sample size and the descriptive nature of the results concerning Aurora-A expression in clinical tumors.

We conclude that AURKA gene is frequently amplified in basal-like breast tumors, and gene amplification frequently leads to its overexpression. Basal-like breast tumors might be good candidates for future cancer therapy with Aurora-A inhibitors and the JIMT-1 cell line may be useful in experimental research with these potential anticancer molecules.

\section{Acknowledgements}

Päivi Myrskyranta, Eeva Riikonen and Sari Toivola are thanked for excellent technical assistance. This study was supported by the Finnish Medical Foundation, The Academy of Finland and Medical Research Fund of Tampere University Hospital.

\section{References}

1. Kallioniemi A, Kallioniemi OP, Piper J, et al: Detection and mapping of amplified DNA sequences in breast cancer by comparative genomic hybridization. Proc Natl Acad Sci USA 91: 2156-2160, 1994.

2. Tanner MM, Tirkkonen M, Kallioniemi A, et al: Independent amplification and frequent co-amplification of three non-syntenic regions on the long arm of chromosome 20 in human breast cancer. Cancer Res 56: 3441-3445, 1996.

3. Sen S, Zhou H and White RA: A putative serine/threonine kinase encoding gene BTAK on chromosome 20q13 is amplified and overexpressed in human breast cancer cell lines. Oncogene 14: 2195-2200, 1997

4. Meraldi P, Honda R and Nigg EA: Aurora kinases link chromosome segregation and cell division to cancer susceptibility. Curr Opin Genet Dev 14: 29-36, 2004.

5. Zhou H, Kuang J, Zhong L, et al: Tumour amplified kinase STK15/BTAK induces centrosome amplification, aneuploidy and transformation. Nat Genet 20: 189-193, 1998.

6. Andrews PD: Aurora kinases: shining lights on the therapeutic horizon? Oncogene 24: 5005-5015, 2005.

7. Fu J, Bian M, Jiang Q and Zhang C: Roles of Aurora kinases in mitosis and tumorigenesis. Mol Cancer Res 5: 1-10, 2007.

8. Harrington EA, Bebbington D, Moore J, et al: VX-680, a potent and selective small-molecule inhibitor of the Aurora kinases, suppresses tumor growth in vivo. Nat Med 10: 262-267, 2004.

9. Soncini C, Carpinelli P, Gianellini L, et al: PHA-680632, a novel Aurora kinase inhibitor with potent antitumoral activity. Clin Cancer Res 12: 4080-4089, 2006.

10. Gautschi O, Heighway J, Mack PC, Purnell PR, Lara PN Jr and Gandara DR: Aurora kinases as anticancer drug targets. Clin Cancer Res 14: 1639-1648, 2008.

11. Johannsson OT, Staff S, Vallon-Christersson J, et al: Characterization of a novel breast carcinoma xenograft and cell line derived from a BRCA1 germ-line mutation carrier. Lab Invest 83: 387-396, 2003. 
12. Tanner M, Kapanen AI, Junttila T, et al: Characterization of a novel cell line established from a patient with Herceptin-resistant breast cancer. Mol Cancer Ther 3: 1585-1592, 2004.

13. Isola J, Tanner M, Forsyth A, Cooke TG, Watters AD and Bartlett JM: Interlaboratory comparison of HER-2 oncogene amplification as detected by chromogenic and fluorescence in situ hybridization. Clin Cancer Res 10: 4793-4798, 2004.

14. Staff S, Isola J and Tanner M: Haplo-insufficiency of BRCA1 in sporadic breast cancer. Cancer Res 63: 4978-4983, 2003.

15. Laakso M, Tanner M, Nilsson J, et al: Basoluminal carcinoma: a new biologically and prognostically distinct entity between basa and luminal breast cancer. Clin Cancer Res 12: 4185-4191, 2006.

16. Ruifrok AC and Johnston DA: Quantification of histochemical staining by color deconvolution. Anal Quant Cytol Histol 23 291-299, 2001.

17. Laakso M, Loman N, Borg A and Isola J: Cytokeratin 5/14 positive breast cancer: true basal phenotype confined to BRCA1 tumors. Mod Pathol 18: 1321-1328, 2005.

18. Marumoto T, Hirota T, Morisaki T, et al: Roles of aurora-A kinase in mitotic entry and G2 checkpoint in mammalian cells. Genes Cells 7: 1173-1182, 2002.

19. Hwang A, Maity A, McKenna WG and Muschel RJ: Cell cycledependent regulation of the cyclin B1 promoter. J Biol Chem 270: 28419-28424, 1995.

20. Wittwer CT, Ririe KM, Andrew RV, David DA, Gundry RA and Balis UJ: The LightCycler: a microvolume multisample fluorimeter with rapid temperature control. Biotechniques 22 : 176-181, 1997.

21. Miyoshi Y, Iwao K, Egawa C and Noguchi S: Association of centrosomal kinase STK15/BTAK mRNA expression with chromosomal instability in human breast cancers. Int J Cancer 92: 370-373, 2001
22. Royce ME, Xia W, Sahin AA, et al: STK15/Aurora-A expression in primary breast tumors is correlated with nuclear grade but not with prognosis. Cancer 100: 12-19, 2004.

23. Reis-Filho JS and Tutt AN: Triple negative tumours: a critical review. Histopathology 52: 108-118, 2008.

24. Tirkkonen M, Johannsson O, Agnarsson BA, et al: Distinct somatic genetic changes associated with tumor progression in carriers of BRCA1 and BRCA2 germ-line mutations. Cancer Res 57: 1222-1227, 1997.

25. Tanner MM, Grenman S, Koul A, et al: Frequent amplification of chromosomal region $20 \mathrm{q} 12-\mathrm{q} 13$ in ovarian cancer. Clin Cancer Res 6: 1833-1839, 2000

26. Bodvarsdottir SK, Hilmarsdottir H, Birgisdottir V, Steinarsdottir M, Jonasson JG and Eyfjord JE: Aurora-A amplification associated with BRCA2 mutation in breast tumours. Cancer Lett 248: 96-102, 2007.

27. Rakha EA, Putti TC, Abd El-Rehim DM, et al: Morphological and immunophenotypic analysis of breast carcinomas with basal and myoepithelial differentiation. J Pathol 208: 495-506, 2006.

28. Neve RM, Chin K, Fridlyand J, et al: A collection of breast cancer cell lines for the study of functionally distinct cancer subtypes. Cancer Cell 10: 515-527, 2006.

29. Tanner MM, Tirkkonen M, Kallioniemi A, et al: Amplification of chromosomal region $20 \mathrm{q} 13$ in invasive breast cancer: prognostic implications. Clin Cancer Res 1: 1455-1461, 1995. 